\title{
Colangiopancreatografía retrógrada endoscópica en una institución de referencia en cáncer
}

\section{Endoscopic retrograde cholangiopancreatography at a leading cancer center}

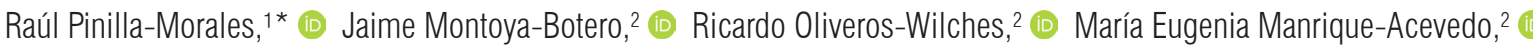

Mario Rey-Ferro, ${ }^{2}$ (1) Mario Abadía-Díaz, ${ }^{2}$ (C) Ricardo Sánchez-Pedraza, ${ }^{3}$ (D) Aurelio de Jesús Garrido-Bohórquez. ${ }^{4}$ (1)

\author{
GacCeso abierto \\ Citación: \\ Pinilla-Morales R, Jaime Montoya-Botero J, \\ Ricardo Oliveros-Wilches R, María Eugenia \\ Manrique-Acevedo ME, Mario Rey-Ferro M, Mario \\ Abadía-Díaz M, Ricardo Sánchez-Pedraza R, \\ Garrido-Bohórquez AJ. Colangiopancreatografía \\ retrógrada endoscópica en una institución \\ de referencia en cáncer. Rev Colomb \\ Gastroenterol. 2020;35(4):430-435. https://doi. \\ org/10.22516/25007440.512
}

Clínica de Gastroenterología. Instituto Naciona de Cancerología. Profesor facultad de medicina Universidad Nacional de Colombia. Bogotá, Colombia.

2 Clínica de gastroenterología, Instituto Nacional de Cancerología. Bogotá, Colombia.

Grupo de Investigación Clínica, Instituto Nacional de Cancerología. Profesor facultad de medicina, Universidad Nacional de Colombia. Bogotá, Colombia.

${ }_{4}^{4}$ Grupo de Investigación Clínica, Instituto

Nacional de Cancerología. Bogotá, Colombia.

*Correspondencia: Raúl Pinilla-Morales. rapinilla@outlook.com

Fecha recibido: $12 / 02 / 20$ Fecha aceptado: $30 / 09 / 20$

\section{Resumen}

Introducción: la endoscopia es una de las herramientas necesarias para el manejo de las enfermedades biliares y pancreáticas. La colangiopancreatografía retrógrada endoscópica (CPRE) es uno de los procedimientos técnicamente más demandantes y de mayor riesgo de complicaciones realizados en el tratamiento de los desórdenes hepatopancreatobiliares. Objetivo: caracterizar los resultados clínicos de los pacientes llevados a CPRE en un centro de pacientes con cáncer exclusivamente. Métodos: estudio de serie de casos observacional, retrospectivo, descriptivo con base en datos clínicos de pacientes llevados a CPRE en una población diagnosticada con cáncer de distintos orígenes primarios y en diferentes estadios de enfermedad desde enero de 2010 hasta enero de 2017. Resultados: se analizaron 255 procedimientos en los que predominó el sexo femenino con 140 casos (54,9\%), el promedio de edad fue 62,9 años (desviación estándar [DE]: 12,9 años). Todos los procedimientos se hicieron con intención terapéutica. La indicación más frecuente fue cáncer de páncreas $(n=47 ; 18,43 \%)$, compresión extrínseca de la vía biliar $(n=42 ; 16,47 \%)$, disfunción de prótesis $(n=36 ; 14,12 \%)$, colecistocoledocolitiasis $(n=32 ; 12,55 \%)$, otras causas $(n=32 ; 12,55 \%)$, cáncer de vesícula ( $n=24 ; 9,41 \%)$, colangiocarcinoma extrahepático $(n=21 ; 8,24 \%)$, coledocolitiasis recidivante $(n$ $=9 ; 3,53 \%)$, coledocolitiasis residual $(n=6 ; 2,35 \%)$ y tumor de Klatskin $(n=6 ; 2,35 \%)$. La canalización exitosa se presentó en 222 casos $(87,06 \%)$. Durante el procedimiento, 10 pacientes presentaron complicaciones $(4,3 \%)$, de las cuales la pancreatitis y las perforaciones fueron las más frecuentes $(n=3 ; 1,18 \%)$, seguidas por sangrado, colangitis $(n=2 ; 0,78 \%)$ y dolor post-CPRE $1(0,39 \%)$. Conclusiones: en la población evaluada, la causa predominante para realizar el procedimiento está relacionada con cáncer y la frecuencia tanto de canalización exitosa como de complicaciones es similar a la de los reportes en las poblaciones generales.

\section{Palabras clave}

Colangiopancreatografía endoscópica, cáncer, centro de referencia.

\section{Abstract}

Introduction: Endoscopy is one of the tools necessary to treat biliary and pancreatic diseases. Endoscopic retrograde cholangiopancreatography (ERCP) is one of the most technically demanding procedures with the highest risk of complications when treating hepato-pancreato-biliary disorders. Objective: To characterize the clinical outcomes of ERCP patients in a cancer patient center. Methodology: Observational, retrospective, descriptive case series study based on clinical data of patients brought to ERCP in a population diagnosed with cancer of different primary origins and in different stages of the disease from January 2010 to January 2017. Results: A total of 255 procedures were analyzed. The female sex was predominant with 140 cases (54.9\%), and the average age was 62.9 years (SD 12.9 years). All procedures were done with therapeutic intent. The most frequent indication was pancreatic cancer $(\mathrm{N}=47 ; 18.43 \%)$, extrinsic compression of the common bile duct $(\mathrm{N}=42$; $16.47 \%)$, biliary prosthesis dysfunction ( $\mathrm{N}=36 ; 14.12 \%)$, cholecystocholedocholithiasis $(\mathrm{N}=32 ; 12.55 \%)$, other causes $(\mathrm{N}=32 ; 12.55 \%)$, gallbladder cancer $(\mathrm{N}=24 ; 9.41 \%)$, extrahepatic cholangiocarcinoma $(\mathrm{N}=21 ; 8.24 \%)$, recurrent choledocholithiasis $(\mathrm{N}=9 ; 3.53 \%)$, residual choledocholithiasis $(\mathrm{N}=6 ; 2.35 \%)$, and Klatskin tumor $(\mathrm{N}=6$; $2.35 \%$ ). Successful cannulation was reported in 222 cases (87.06\%). During the procedure, 10 patients presented complications (4.3\%), with pancreatitis and perforations being the most frequent $(\mathrm{N}=3 ; 1.18 \%)$, followed by bleeding, cholangitis ( $\mathrm{N}=2 ; 0.78 \%)$, and post-ERCP pain ( $\mathrm{N}=1 ; 0.39 \%)$. Conclusions: In the population studied, the principal reasons for performing the procedure were related to cancer. The frequency of successful cannulation and complications reported here are similar to other results in the general population.

\section{Keywords}

Endoscopic cholangiopancreatography, cancer, reference center. 


\section{INTRODUCCIÓN}

Las enfermedades del tracto biliar y del páncreas frecuentemente requieren de procedimientos endoscópicos como parte de su manejo. La colangiopancreatografía retrógrada endoscópica (CPRE) ha jugado un papel importante en el tratamiento de enfermedades benignas y malignas desde su introducción en $1968(1,7)$; a pesar de que en un principio las imágenes de colangiografía obtenidas por endoscopia brindaron nuevas posibilidades diagnósticas de las enfermedades del árbol biliar y del páncreas, desde hace varios años, con el desarrollo de estudios no invasivos como la resonancia magnética y la ultrasonografía endoscópica, el papel predominante de la CPRE es terapéutico, evitando así exploraciones netamente diagnósticas y con esto la posibilidad de complicaciones derivadas del procedimiento.(2).

Las neoplasias originadas en el árbol biliar y el páncreas, así como otros tumores digestivos e incluso aquellos originados en otros órganos pueden ocasionar obstrucción biliar tanto por el compromiso localmente avanzado como por la enfermedad metastásica y, de esta forma, requerir intervenciones terapéuticas endoscópicas para el alivio la obstrucción biliar y las consecuencias de esta. Así mismo, los pacientes con cáncer no son ajenos a presentar complicaciones de la enfermedad litiásica biliar.

Los centros que exclusivamente se encargan del manejo del cáncer usualmente cuentan con servicios de endoscopia digestiva de alta complejidad que realizan el diagnóstico y el tratamiento preoperatorio o paliativo de los distintos tumores digestivos que afectan la vía biliar (3).

La CPRE es un procedimiento endoscópico especial en el cual se abordan las vías biliar y pancreática con la intención de confirmar situaciones que requieran algún manejo como extracción de cálculos, toma de tejido y restitución del flujo biliar por medio de la colocación de prótesis plásticas o metálicas autoexpandibles. El procedimiento se lleva a cabo bajo una guía fluoroscópica y sedación o anestesia general; existen riesgos de complicaciones, las cuales pueden corresponder a las que ocurren con cualquier procedimiento endoscópico y las específicas de la intervención, dentro de las cuales se describen pancreatitis aguda (que es la más común), hemorragia, perforación, colangitis, dolor pos-CPRE e impactación de canastilla, entre otros. Las diferentes series describen la ocurrencia de complicaciones hasta en un $10 \%$ de los casos y el procedimiento tiene una mortalidad hasta el $1 \%$ (4).

Están bien descritos los factores de riesgo para la presentación de complicaciones como aquellos asociados con el procedimiento, dentro de los cuales el adecuado entrenamiento de quien lo realiza juega un papel preponderante, así como factores específicos del paciente y la asociación entre estos determinarán la aparición de dichos eventos $(5,6)$.

La presente investigación se realiza con el fin de caracterizar las CPRE realizadas en los pacientes del Instituto Nacional de Cancerología intervenidos entre enero de 2010 y enero de 2017, los cuales tienen patología oncológica ya sea del árbol pancreatobiliar o fuera de este, con el fin de caracterizar los resultados de esta intervención.

\section{MATERIALES Y MÉTODOS}

El presente es un estudio observacional, retrospectivo, descriptivo de serie de casos. Se extrajo la información por medio de la revisión de las historias clínicas de pacientes sometidos a CPRE en el servicio de cirugía gastrointestinal y endoscopia digestiva del Instituto Nacional de Cancerología de Bogotá a partir el mes de enero de 2010 hasta el mes enero de 2017. En caso de no encontrar la información, se realizó contacto telefónico con el paciente a través del número consignado en la misma.

Los procedimientos fueron realizados por especialistas en cirugía gastrointestinal, gastroenterología y endoscopia digestiva con más de 150 casos al año y en algunas ocasiones con la participación de especialistas en entrenamiento, se llevaron a cabo bajo anestesia general y se realizaron con videoduodenoscopio Olympus TJF-145. Se registraron las características demográficas y clínicas con respecto a la indicación del procedimiento, así como la frecuencia de complicaciones durante y después del procedimiento de cada uno de los pacientes mayores de 18 años, a los que se les realizó el procedimiento en mención en el Instituto Nacional de Cancerología durante el período del estudio y que contaron con un seguimiento de al menos 30 días. La información relacionada con la medición de variables fue grabada en una base de datos diseñada para tal fin en el programa Access ${ }^{\circ}$; esa base permitió administrar las variables que fueron exportadas al programa Stata $12^{\circledR}$ para el respectivo análisis. El proyecto fue aprobado y seguido por el comité de ética en investigación del Instituto Nacional de Cancerología.

\section{Análisis estadístico}

El enfoque utilizado en el análisis fue descriptivo. Las variables categóricas fueron resumidas como porcentajes. En el caso de las variables continuas, se usaron como medida de resumen medias y desviaciones estándar (DE) o medianas junto con el correspondiente rango intercuartílico (RIC). Como desenlace, se consideró la muerte relacionada con el procedimiento; para su descripción se estimaron tasas de densidad de incidencia, teniendo en cuenta el tiempo diferencial de seguimiento. Estas tasas se expresaron tomando 
como denominador 1000 pacientes por mes y se calcularon tomando las pérdidas de seguimiento o la no presencia de desenlace como censuras a la derecha; las tasas se calcularon junto con sus correspondientes intervalos de confianza (IC) del $95 \%$.

\section{RESULTADOS}

Durante el período del estudio se realizaron en total 255 CPRE para el tratamiento de patologías del árbol hepatopancreatobiliar. Las características sociodemográficas y clínicas de los pacientes se describen en la Tabla 1.

Tabla 1. Características sociodemográficas y clínicas de los pacientes a quienes se les realizó CPRE

\begin{tabular}{|c|c|}
\hline Variable & n (\%) \\
\hline Procedimientos & 255 \\
\hline Sexo femenino & $140(54,9)$ \\
\hline \multicolumn{2}{|l|}{ Régimen de salud } \\
\hline - Contributivo & $139(54,5)$ \\
\hline - Subsidiado & $116(45,5)$ \\
\hline Comorbilidades & $132(51.8)$ \\
\hline - Cardiopatía & $88(34,51)$ \\
\hline - Neumopatía & $4(1,57)$ \\
\hline - Nefropatía & $2(0,78)$ \\
\hline - Neuropatía & $3(1,18)$ \\
\hline $\begin{array}{l}\text { Otras enfermedades (diabetes, anticoagulación, } \\
\text { hepatitis crónica, obesidad mórbida, VIH, } \\
\text { epilepsia, TVP, hepatopatía) }\end{array}$ & $35(13,73)$ \\
\hline ASA III & $129(50,59)$ \\
\hline Peso (RIC) & $59(14)$ \\
\hline Talla (RIC) & $160(13)$ \\
\hline
\end{tabular}

ASA: escala American Society of Anesthesiologists; TVP: trombosis venosa profunda; VIH: virus de inmunodeficiencia humana.

Se realizaron más intervenciones en las pacientes de sexo femenino; la media de la edad fue 62,9 años (DE: 12,9 años). Aproximadamente, la mitad de los pacientes presentó algún tipo de comorbilidad, de las cuales la más frecuente fue la cardiopatía. El índice de masa corporal (IMC) tuvo una media de $22 \mathrm{~kg} / \mathrm{m}^{2}$ para los 192 pacientes. Todas las CPRE realizadas fueron con intención terapéutica.

Las indicaciones de realización del procedimiento más frecuentes fueron cáncer de páncreas, compresión extrín-

seca de la vía biliar, disfunción de prótesis y colecistocoledocolitiasis (Tabla 2). La compresión de la vía biliar tuvo como causas neoplásicas más frecuentes los linfomas, cáncer de mama y de cérvix, y seminomas testiculares con compromiso retroperitoneal. Dentro de otras causas se describen tumores periampulares, páncreas divisum, fístula biliar, fístula pancreática y estenosis del colédoco. Solo dos procedimientos se realizaron en pacientes que tenían alteración en la anatomía del tracto gastrointestinal por antecedente de gastrectomía subtotal debido a cáncer de estómago y reconstrucción con gastroyeyunostomía en asa, lo que permitió abordar la papila con un duodenoscopio convencional realizando papilotomía sobre un stent plástico $\left(\right.$ Flexima $\left.^{\mathrm{TM}}\right)$ de $7 \mathrm{~cm}$ por $7 \mathrm{Fr}$ con bisturí de aguja $\left(\right.$ MicroKnife $\left.^{\mathrm{TM}}\right)$, con la posterior extracción de cálculos (Figura 1).

Tabla 2. Indicaciones de realización del procedimiento

Tipo de indicación

\begin{tabular}{lc|}
\hline Cáncer de páncreas & $47(18,43)$ \\
\hline Compresión extrínseca & $42(16,47)$ \\
\hline Disfunción de prótesis & $36(14,12)$ \\
\hline Colecistocoledocolitiasis & $32(12,55)$ \\
\hline Tumores periampulares & $21(8,23)$ \\
\hline Otros & $11(4,32)$ \\
\hline Cáncer de vesícula & $24(9,41)$ \\
\hline Colangiocarcinoma extrahepático & $21(8,24)$ \\
\hline Coledocolitiasis recidivante & $9(3,53)$ \\
\hline Coledocolitiasis residual & $6(2,35)$ \\
\hline Tumor de Klatskin & $6(2,35)$
\end{tabular}

La mayoría de las intervenciones (190) se realizó con el paciente hospitalizado $(74,51 \%)$. Durante las intervenciones, solo 13 procedimientos $(5,1 \%)$ requirieron papilotomía de precorte. Con respecto al uso de prótesis, se observó una inserción mayor de las plásticas $(\mathrm{n}=127 ; 49,80 \%)$ y metálicas $(\mathrm{n}=13 ; 5,10 \%)$; solo se liberaron 7 prótesis pancreáticas (2,75\%). En 46 de las intervenciones (18,04\%) se extrajeron cálculos biliares y en un ningún caso se utilizó litotricia mecánica. De los pacientes con tumores periampulares, a 5 (1,9\%) se les realizó ampulectomía durante el procedimiento. Se logró la canalización exitosa en 222 intervenciones (87,06\%), de las cuales en 209 (81,96\%) se logró el éxito terapéutico. Durante el procedimiento, 10 pacientes presentaron complicaciones, de las cuales la pancreatitis y las perforaciones fueron las más frecuentes (Tabla 3). 

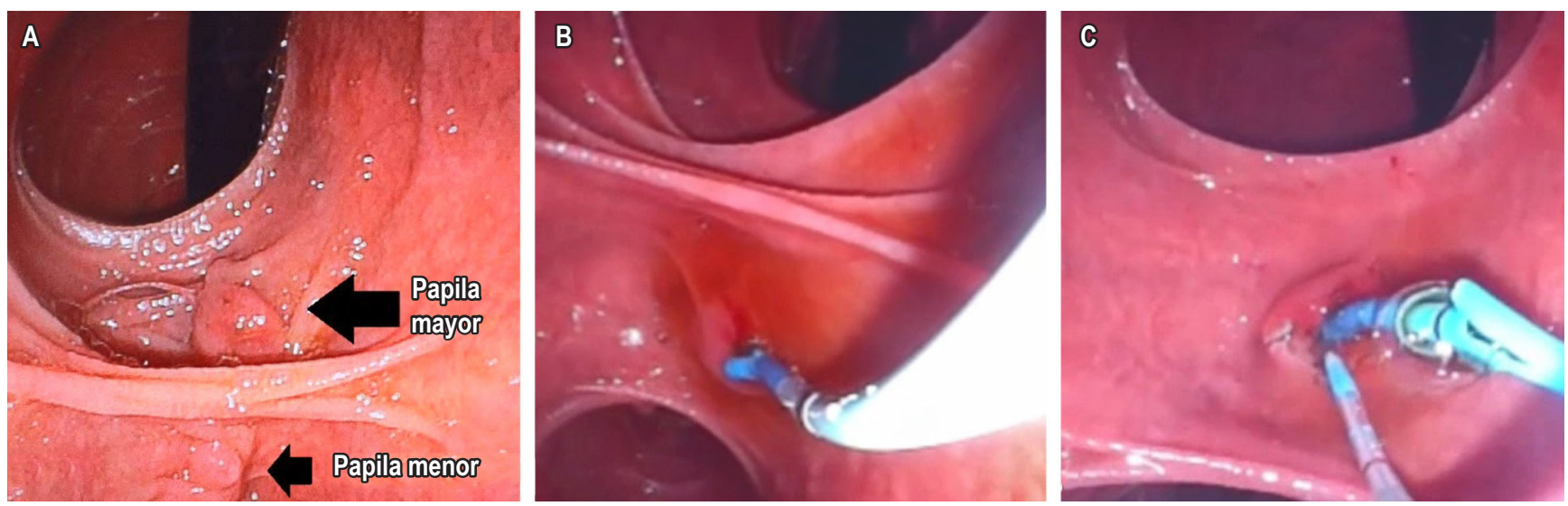

Figura 1. A. Visión de la región papilar desde el abordaje retrógrado. B. Colocación de un stent plástico temporal. C. Realización de papilotomía con bisturí de aguja sobre el stent.

Tabla 3. Complicaciones de la CPRE

\begin{tabular}{|lc|}
\hline \multicolumn{1}{c}{ Complicación } & n (\%) \\
\hline Complicaciones (total) & $10(4)$ \\
\hline Pancreatitis pos-CPRE & $3(1,1)$ \\
\hline Sangrado & $2(0,78)$ \\
\hline Perforación & $3(1,1)$ \\
\hline Colangitis & $2(0,78)$ \\
\hline Dolor pos-CPRE & $1(0,39)$ \\
\hline Eventos adversos de medicamentos & 0 \\
\hline Cardiovasculares & 0 \\
\hline Impactación de canastilla & 0
\end{tabular}

El análisis de mortalidad relacionada se efectuó con 240 observaciones (fueron excluidos del análisis 15 pacientes debido a que no tuvieron disponible información sobre el seguimiento). Los 240 pacientes aportaron un total de 880,8 meses de seguimiento (tiempo mínimo de seguimiento: 0,03 meses; tiempo máximo de seguimiento: 98,5 meses). En ese período se documentaron dos muertes relacionadas con la CPRE. Esto supone una tasa de mortalidad relacionada con el procedimiento de 2,3 muertes por cada 1000 pacientes/ mes (IC 95 \%: 0,57 a 9,1). Dada la baja frecuencia de eventos, no fue estimable la mediana de supervivencia.

\section{DISCUSIÓN}

La CPRE terapéutica con esfinterotomía para la extracción de cálculos de la vía biliar, colocación y recambio de stents biliares y pancreáticos es uno de los procedimientos más desafiantes en la endoscopia digestiva. El éxito depende del entrenamiento, habilidad y experiencia del endoscopista, y la tasa de éxito varía entre $90 \%$ y $95 \%$ cuando es realizada por expertos (8).

La frecuencia de comorbilidades y la distribución por sexo en el presente estudio mostraron similitud con lo publicado en otros reportes (8-12).

Las indicaciones más frecuentes fueron el cáncer de páncreas y la compresión extrínseca de la vía biliar. En contraste con lo reportado en la literatura, la colecistocoledocolitiasis tuvo una frecuencia de solo el 12,55 \% (13). Todos los procedimientos se realizaron con intención terapéutica y se logró la canalización de la vía biliar exitosa en el 87,06 \% con drenaje biliar adecuado o extracción de cálculos según la indicación en el 81,96\% de los casos.

Se realizaron 5 ampulectomías en los pacientes de la muestra. Los pacientes a quienes se les implantó un stent plástico presentaron una mediana de 3 semanas para sufrir disfunción y se requirió de 8 semanas para el cambio a prótesis metálica autoexpandible. Como se mencionó previamente, las complicaciones más frecuentes fueron la pancreatitis después del procedimiento y la perforación; la primera complicación tuvo una frecuencia menor que la incidencia publicada, la cual alcanza hasta el $4 \%(8,10)$, y los cuadros clínicos se resolvieron el con manejo médico; y en cuanto a la segunda complicación, uno de los casos se presentó a nivel del antro y se resolvió de forma inmediata con la colocación de un clip tipo OVESCO con la posterior finalización del procedimiento; mientras que los otros dos casos, catalogados como extravasación del medio posterior a papilotomía e interpretados como lesiones del conducto biliar, se manejaron con un stent biliar plástico 
sin desarrollo de síntomas luego del procedimiento; dicha frecuencia y manejos son similares a lo reportado en la literatura $(0,08 \%$ a $1,6 \%)(14)$. Se presentó sangrado en un menor número de pacientes, de los cuales uno falleció; sin embargo, el origen del sangrado fue atribuido a várices esofágicas. Un paciente presentó dolor pos-CPRE que se resolvió al siguiente día, luego de haber descartado pancreatitis y perforación; sin embargo, al ser un estudio retrospectivo no puede descartarse la posibilidad de subregistro de algunas de estas variables en los registros clínicos (15).

Ninguno de nuestros pacientes presentó complicaciones relacionadas con medicaciones, eventos cardiovasculares o dispositivos implantados; dichas complicaciones son poco reportadas en la literatura (15).

En nuestra experiencia como centro exclusivo para el manejo del cáncer en Colombia, respecto al abordaje endoscópico de la vía biliar en pacientes que no solamente presentan neoplasias del árbol hepatopancreatobiliar, contamos con una tasa de éxito de las intervenciones de acuerdo con la experiencia mundial y la tasa de complicaciones es similar a la reportada en la literatura, en centros con mayor número de pacientes probablemente explicado por el volumen de procedimientos realizado por cada uno de los especialistas en otras instituciones (16). La principal limitación del presente estudio es su carácter retrospectivo, así como la dificultad para el seguimiento de los pacientes por condiciones netamente de aseguramiento, lo que también podría explicar el número reducido de casos a pesar de encontrarnos en un centro de manejo exclusivo de pacientes con cáncer. El hecho de que la mayor parte de las variables se recuperan de información consignada en los registros clínicos es una limitación propia de los estudios retrospectivos, ya que puede ser una fuente importante de sesgos.

\section{CONCLUSIONES}

En una institución que maneja exclusivamente pacientes con cáncer, la causa predominante para realizar la CPRE está relacionada predominantemente con neoplasias digestivas que ocasionan obstrucción biliar; así mismo, la litiasis es más escasa. La frecuencia de canalización exitosa y la de complicaciones del procedimiento son similares a las reportadas en poblaciones que manejan enfermedades benignas y malignas.

\section{Agradecimientos}

A todo el personal administrativo y asistencial del servicio de gastroenterología oncológica del Instituto Nacional de Cancerología.

\section{Fuente de financiación}

Instituto Nacional de Cancerología.

\section{REFERENCIAS}

1. McCune WS, Shorb PE, Moscovitz H. Endoscopic cannulation of the ampulla of vater: a preliminary report. Ann Surg. 1968;167(5):752-6. https://doi.org/10.1097/00000658-196805000-00013

2. NIH state of the science statement on endoscopic retrograde cholangiopancreatography (ERCP) for diagnosis and therapy. NIH Consens State Sci Statements. 2002;19(1):1-26.

3. Kutty RS, Westaby D, Martin JP, Vlavianos P, Andreyev HJN. What are the Gastrointestinal Endoscopic Requirements of a Cancer Centre? Clin Oncol (R Coll Radiol). 2007;19(5):330-2. https://doi.org/10.1016/j.clon.2007.03.008

4. Freeman ML. Adverse outcomes of endoscopic retrograde cholangiopancreatography: avoidance and management. Gastrointest Endosc Clin N Am. 2003;13(4):775-98, xi. https://doi.org/10.1016/S1052-5157(03)00107-7

5. Baillie J. Endoscopic therapy in acute recurrent pancreatitis. World J Gastroenterol. 2008;14(7):1034-7. https://doi.org/10.3748/wjg.14.1034
6. Donnellan F, Byrne MF. Prevention of Post-ERCP Pancreatitis. Gastroenterol Res Pract. 2012;2012:796751. https://doi.org/10.1155/2012/796751

7. Kawai K, Akasaka Y, Murakami K, Tada M, Koli Y. Endoscopic sphincterotomy of the ampulla of Vater. Gastrointest Endosc. 1974;20(4):148-51. https://doi.org/10.1016/S0016-5107(74)73914-1

8. Baillie J. ERCP training: for the few, not for all. Gut. 1999;45(1):9-10. https://doi.org/10.1136/gut.45.1.9

9. Vandervoort J, Soetikno RM, Tham TC, Wong RC, Ferrari AP Jr, Montes H, Roston AD, Slivka A, Lichtenstein DR, Ruymann FW, Van Dam J, Hughes M, Carr-Locke DL. Risk factors for complications after performance of ERCP. Gastrointest Endosc. 2002;56(5):652-6. https://doi.org/10.1067/mge.2002.129086

10. ASGE Standards of Practice Committee, Chandrasekhara V, Khashab MA, Muthusamy VR, Acosta RD, Agrawal D, Bruining DH, Eloubeidi MA, Fanelli RD, Faulx AL, Gurudu SR, Kothari S, Lightdale JR, Qumseya BJ, 
Shaukat A, Wang A, Wani SB, Yang J, DeWitt JM. Adverse events associated with ERCP. Gastrointest Endosc. 2017;85(1):32-47. https://doi.org/10.1016/j.gie.2016.06.051

11. Kochar B, Akshintala VS, Afghani E, Elmunzer BJ, Kim KJ, Lennon AM, Khashab MA, Kalloo AN, Singh VK. Incidence, severity, and mortality of post-ERCP pancreatitis: a systematic review by using randomized, controlled trials. Gastrointest Endosc. 2015;81(1):143-149.e9. https://doi.org/10.1016/j.gie.2014.06.045

12. Shanmugarajah I, Solhaug M, Aslam O, Reiertsen O. Efficacy and safety assessment of ERCP in patients with malignant biliary obstruction. Acta Gastroenterol Belg. 2017;80(4):487-91.

13. Cohen ME, Slezak L, Wells CK, Andersen DK, Topazian $\mathrm{M}$. Prediction of bile duct stones and complications in gallstone pancreatitis using early laboratory trends. Am J Gastroenterol. 2001;96(12):3305-11.

https://doi.org/10.1111/j.1572-0241.2001.05330.x
14. Stapfer M, Selby RR, Stain SC, Katkhouda N, Parekh D, Jabbour N, Garry D. Management of duodenal perforation after endoscopic retrograde cholangiopancreatography and sphincterotomy. Ann Surg. 2000;232(2):191-8. https://doi.org/10.1097/00000658-200008000-00007

15. Penaloza-Ramirez A, Leal-Buitrago C, RodriguezHernandez A. Adverse events of ERCP at San Jose Hospital of Bogota (Colombia). Rev Esp Enferm Dig. 2009; 101(12):837-49. https://doi.org/10.4321/S1130-01082009001200003

16. Peng C, Nietert PJ, Cotton PB, Lackland DT, Romagnuolo J. Predicting native papilla biliary cannulation success using a multinational Endoscopic Retrograde Cholangiopancreatography (ERCP) Quality Network. BMC Gastroenterol. 2013;13:147. https://doi.org/10.1186/1471-230X-13-147 